\title{
Designing Personal Informatics for Self-Reflection and Self-Awareness: the Case of Children with Attention Deficit Hyperactivity Disorder
}

\author{
Juan Jimenez Garcia, Hilde de Bruyckere, David Keyson, Natalia \\ Romero \\ Delft University of Technology, Department of Industrial Design Engineering, ID- \\ StudioLab, Delft, The Netherlands \\ \{j.c.jimenezgarcia,d.keyson,n.a.romero\}@tudelft.nl,h_debruyckere@hotmail.com
}

\begin{abstract}
A main challenge in designing for children with Attention Deficit Hyperactivity Disorder (ADHD) is to support the learning process of supressing undesired behaviour on daily routines by means of positive feedback and rewards. Personal Informatics (PI) is a model that supports capturing and integration of personal data to facilitate reflection and action that is used as a design platform to support behavioral learning. This paper presents a designdriven research study that illustrates the potential of PI to support selfawareness and self-reflection of ADHD children. Two design approaches are described which aim to support self-behavioral inhibition learning: (a) KITA, a Kinesiofeedback Toy for ADHD, being a Tangible User Interface that measures and assesses children's activity and provides them with feedback as to whether or not behavior is within appropriate limits; and (b) WRISTWIT, a Wearable device presenting information on attention and time for ADHD to increase ontask behavior. KITA and WRISTWIT were tested in the field with children as design means to implement PI to positively modify children behavior during daily school routines.
\end{abstract}

Keywords: Personal Informatics, children, Attention Deficit Hyperactivity Disorder, ADHD, Tangible User Interface, wearable, self-awareness, selfreflection, Empirical Research Through Design.

\section{Introduction}

ADHD is the most commonly diagnosed behavioral childhood disorder affecting $4 \%$ to $7 \%$ of children worldwide [1,2]. The main symptoms of ADHD are hyperactivity, inattention and lack of self-control [3]. When working in a class with little personalized supervision, children with ADHD easily loose their attention, start moving more, and stop working [4,5]. With their inability to focus for long periods and the need for stimilu, ADHD children rely on others to support positive behavior by providing external motivation [5]. When their attention has shifted they are often unable to get back to work without an external source telling or reminding them to do it [6].Therefore, the feedback they require has to be delivered at the moment in time 
the behavior is present, the point of performance. This makes it hard to treat since therapists, parents and teachers cannot be at each point of performance [5] and it also causes limitations in their independency. Two main behavioral aspects of ADHD that affect school periods were taken as the starting points to explore the role of Personal Informatics (PI) in supporting self-awareness and self-reflection for ADHD children.

The first aspect is related to the motor excess, as the most visible symptom, it is often the first indication parents and teachers detect in children. Over-activity is considered the most disrupting symptom in school setting. It affects their ability to focus on daily life activities, appointments, and goal-accomplishment, which could lead to social problems at home and with peers [1]. ADHD children express their emotions physically by showing unconscious high levels of arousal and energy expenditure. This characteristic requires behavioral interventions and medical therapies to decrease the symptoms. However, current solutions do not encourage children to learn how to self-control in a playful way $[7,8,9]$. As a result, many of these concepts are impersonal, and not particularly suitable for children to improve their learning experience of self-control and self-monitoring.

The second area focuses on the perception of time where a faulty sense of time awareness and management is seen as one of the underlying symptoms in ADHD [4]. As Barkley states, self-awareness across time is the ultimate yet nearly invisible disability afflicting ADHD individuals [4]. A deficit in time perception is also mentioned by Yang et al. (2007), suggesting that children with ADHD have a faster sense of time internally [10]. Therefore, future goals do not guide their present behavior, leaving them easily distracted by more immediate satisfaction in the here and now when a situation does not provide the immediate consequences or rewards. ADHD children are unable to estimate time spans and seem to feel no clear difference between small and large amounts of time. During classes where the child has to work independently this behavior is more evident. Their uncertainty about time, makes it difficult for them to independently adjust their behavior [10] ADHD children need support on-task behavior as well as support to get their attention back to class when being distracted. Motivation needs to be provided to increase their attention during the whole class.

Personal Informatics (PI) is an emerging area in the field of Human-Computer Interaction that facilitates people to collect and visualize personal relevant information for the purpose to support self-reflection and action for behavioral change [11]. Despite the importance of self-reflection in PI systems, most of the studies to date have been focused on supporting collection and representation of data with the implementation of new automated sensors, tools and attractive visualizations. In line with $[12,13]$, the challenge in PI is the design of meaningful data towards in-depth self-awareness and self-reflection. Based on a review of current PIs, there are no systems that address the needs of children with specific health-related conditions such as Attention Deficit Hyperactivity Disorder in a specific context, such as during daily routines at school.

The following sections describe KITA and WRISTWIT as design studies, which were aimed at exploring the potential of collecting and displaying personal data to support ADHD children at school by facilitating self-awareness and self-reflection on-task behavior. The design and test of these prototypes were conducted by means of the Empirical Research Through Design (ERTD) process [14] in which design 
concepts were evaluated with teachers, parents and children to define the functional characteristics and the scope of the solutions through iterative design prototypes, which were tested with specific research variables embedded in the designs.

The design experiences described in this paper aims to inform the potential of designing and implementing supportive devices for ADHD, as well as to contribute to Personal Informatics by: (1) extending the challenge in developing devices that focus on the reflection stage as a core source for users to take action towards self-behavior modification, and (2) to provide insights in the design process of such systems with different prototypes iterations tested in-situ for health-related contexts.

\section{KITA}

Kinesiofeedback Toy for ADHD (KITA) is a behavioral technology device that aims to improve self-monitoring and self-control of kinetic behavior for children between 4-7 years old. Based on insights gained from context mapping techniques [15], observation sessions and mock-ups tested with children, it was found that they are emotionally attached to animals as friendly companions. Therefore, the final prototype is inspired by the design of a creature-like Tangible User Interface (TUI) (Fig 1a). KITA measures physical activity levels using an accelerometer and provides vibration feedback to inform the child that a predefined activity level threshold has been exceeded. A built-in agenda is used to adjust the threshold depending if it is class or break time. Monitoring of activity levels is reinforced during class time in form of a smiley face (Fig. 1b).

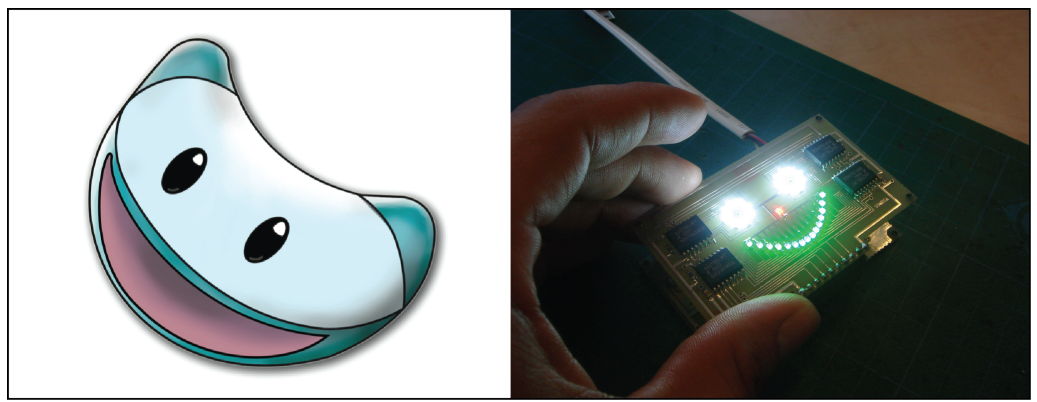

Fig. 1. KITA a) Cartoon-character concept; b) Prototype

\subsection{Design and Implementation}

Four main design phases led to the development of KITA. In the first phase, on-site observations of children with ADHD were conducted at a regular and a special school for ADHD children in Delft, The Netherlands. In both schools first, second, and third grade classes were observed. Children ranged in age from four to seven. At the special school De Bouwsteen Librijn, observations provided insights on how children and caregivers dealt with ADHD symptoms during class hours and breaks, while the 
regular school, De Horizon School, provided comparative information about how normal kids behave. Additionally, nine interviews were conducted with 2 specialists, 4 parents and 3 teachers at the special school, and with the teachers of the regular school. Later, a brainstorming session with 2 teachers and 3 design colleagues was conducted. The ideas from participants were helpful to open the possibilities of a toy/game in a form of "the perfect game for ADHD". Three design concepts were finally chosen and developed further through an iterative design process.

The third phase of development involved the test in laboratory of these design concepts with three ADHD children by means of three mock-ups. Each mock-up was related to a pre-defined game in which children had to accomplish specific tasks. The results provided a definition of the functional and non-functional parameters of a final design concept as follows: a) provide time out, reward desired behavior; b) support scheduled activities such as class time and play time; c) support real-time progress feedback; d) use a creature-like toy as a design concept; and e) a friendly companion wear it on the waist.

The fourth phase focused on defining behavior thresholds based on the data provided by an accelerometer. In order to define class-time related thresholds, a prototype with a 3-axis accelerometer was developed to support explorations at school (Fig. 2). Children used the device on their waist in the classroom while they were listening to the teacher and during self-study. This prototype was additionally used to define playtime related thresholds. A game was arranged with 10 children in the playground to observe the maximum levels they could get while playing with the device on their waist. Along with observations, the data analysis suggested kinetic behavior boundaries that were used as parameters to deliver feedback. The used of a 3 -axis accelerometer assesses the body activity by registering the counts of movements in $\mathrm{X}, \mathrm{Y}, \mathrm{Z}$ axes. These counts depend on the duration and intensity of a single movement.
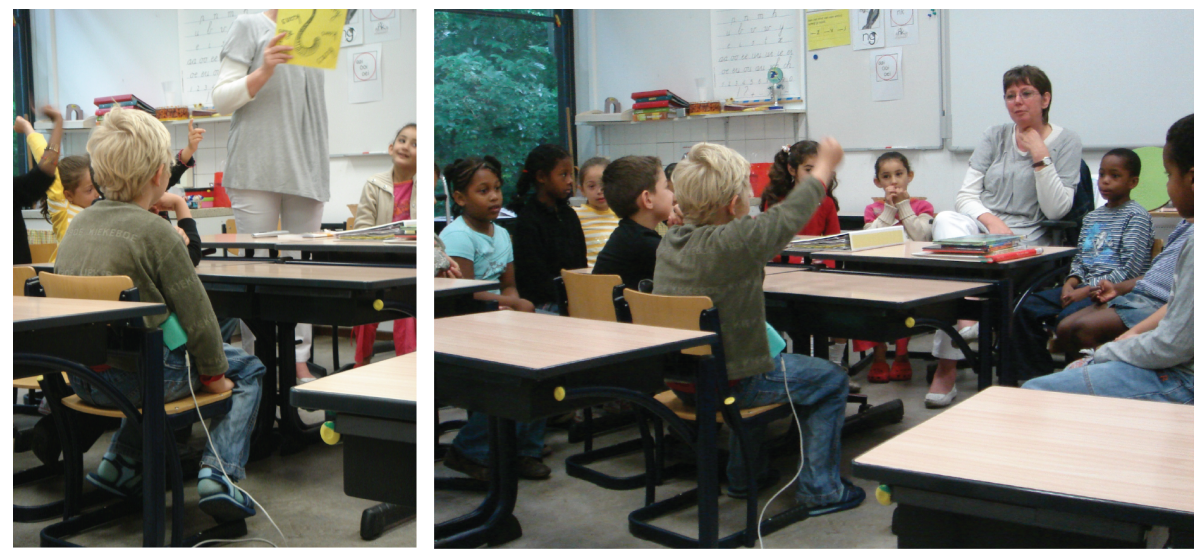

Fig. 2. Initial prototype. Collecting inertial data to define kinetic behavior thresholds during class time 


\subsection{Final Concept and Prototype}

The toy consists of two main parts: KITA, the creature companion, removable and portable (Figure 3), that aims to support self-control and self-monitoring by providing feedback on behavior progress; and its nest, a ball (Figure 3), addresses self-reflection by providing dynamic rewards.

After KITA' battery has been charged all night while placed in its nest, the child removes KITA from the ball and takes it to school. If the child wants to play with KITA afterwards, he needs to take care of it by letting KITA sleeps during class time. In this way, KITA will be happy and rested enough to play. When the device detects excess body behavior during class time vibration feedback is provided. Whether the child exceeds the defined number of activity counts during a preset time interval, or he remains in the defined activity threshold, KITA communicates the event to the child by means of its changing moody face. KITA's face involves two main parts: a) the eyes that help the child to be aware when is class time (sleeping state) and play time (awake state); and b) the smile that represents how the good/bad behavior of the child progresses overtime at school. The better the child behaves the wider is the KITA's smile. The visualization of the data as a smiley face facilitates selfmonitoring on the go. The final prototype comprises in total 31 LEDs to represent KITA's face, a vibration motor, a rechargeable battery, a 3-axis accelerometer and an IR sensor to detect when KITA is placed in the ball to transfer the collected data to the ball (Fig. 3a).

Later at home, the child can place KITA in its nest. The ball reacts accordingly to the data assessed by KITA during the day. As a reward, the child is able to play with it using KITA's nest. The ball provides sounds and light feedback showing how happy KITA is after school (Fig. 3b). The amount of playtime is based on the assessment of kinetic behavior performed during school time. It is stated that ADHD children lack in bringing their over-arousal to normal levels when they have started an exciting activity [33]. Therefore, after playing for a while, KITA communicates to the child that it is getting tired, encouraging him to cool-down along, thus, reducing his arousal level. This feedback, enabled by a programmed microcontroller, aims to tutor the child to stop at the right time. KITA's nest comprises the micro-controller, a speaker, 8 LEDs, an IR sensor and a rechargeable battery.

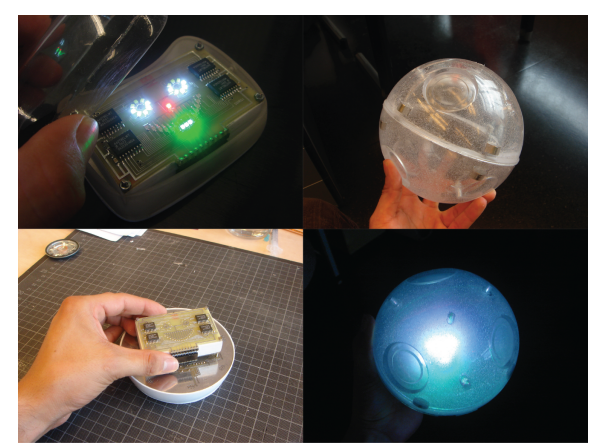

Fig. 3. KITA's main components: a) Left. KITA's face expressions and nest b) Right: rubber ball containing KITA's nest and providing sound and light feedback 


\subsection{Test and Summary of the Results}

To evaluate how KITA elicited the intended behavior, two days of baseline measurement, were followed by two days of intervention with two ADHD children. In the baseline condition, the KITA display was concealed and measurements of children physical activity patterns were taken during class time (Fig. 4a). Raw data was collected registering how many peaks of high activity were detected during class time, in relation to the smile progress of KITA after each class session and the final smile at the end of the school day. Results of baseline and intervention were compared to detect behavioral change in relation to the displayed smile progress in KITA. Although the pilot test was short to draw conclusive results, initial findings showed an interesting trend in which children's activity level in class was reduced by $16 \%$ when using KITA.

After the tests, interviews were conducted with 2 teachers and the children that participated in the test to get insights about how they felt when using the prototype. Children reported to feel attached and curious about making KITA happier. Although children felt proud of having such a toy during class, further research is need to investigate to what degree the sense novelty of the toy may decrease over time, affecting children's sense of attachment with KITA.
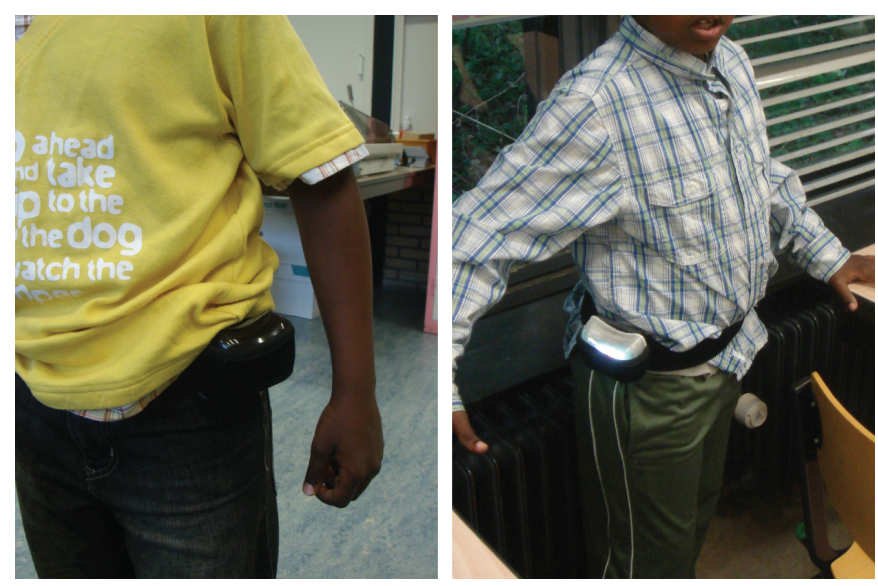

Fig. 4. a) Left, baseline and b) right, intervention. The device featured two different shields to hide and expose KITA's feedback capabilities

\section{WRISTWIT}

WRISTWIT is a wearable device targeted at ADHD children between 8-12 years old to improve their ability to work independently by supporting sense of time and attention in classes, where the environment cannot provide this much needed support. With insights gained from context mapping techniques [15] observations and a focus group with children, the design concept resulted into a personal bracelet that provides 
information to the child. WRISTWIT is designed to increase on-task behavior by: (1) increasing the sense of time, the bracelet displays time progress in a low-complex visual representation of 60 minutes by using 12 LED lights, each LED representing 5 minutes; and (2) increasing the level of attention, the device monitors body movement by means of an accelerometer alerting the child when he or she is inattentive.
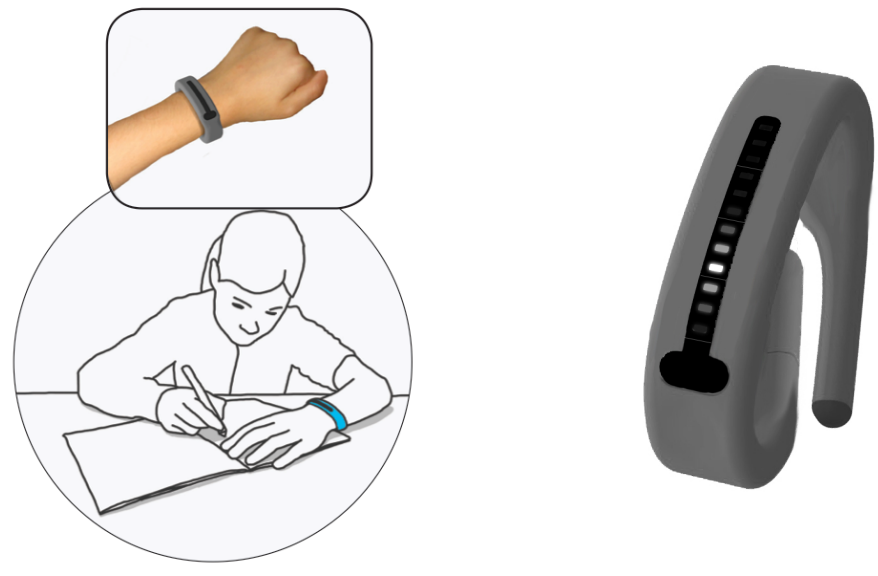

Fig. 5. WRISTWIT. Bracelet in use

\subsection{Design and Implementation}

Exploring time perception in ADHD children. The final concept of WRISTWIT was preceded by 2 explorative phases. The first phase consisted of observations and interviews that took place at two schools in The Netherlands to understand how ADHD behavior is specific to time in the school context. In total 7 observation sessions of 1 to 3 hours took place and 15 qualitative interviews were conducted with 8 teachers, 4 experts and 5 parents, giving the possibility to pose questions that arose during observations. Observations started at a regular elementary school in Delft, at the De Oostpoort. Two groups of children (age 8-10) and two groups (age 10-12) were observed during times in which at least one hour of independent study was required. Next, observations took place at a special elementary school, De Elsenburgschool, in Rijswijk for children with learning disabilities where 10-20\% of children are diagnosed with ADHD. The researchers attended a regular class day with three groups with ages ranging from 8-9, 9-11 and 10-12. Later, in order to receive more in-depth information on time, attention and motivation, a focus group was conducted with 5 children. The qualitative information provided by sensitizing booklets used during the session was analyzed and categorized in quotes and themes with concluding statements that helped to define the requirements for WRISTWIT. When looking over the resulted insights, the need to help student's behavior by providing a sense of time and help focus attention was evident here. It was also found that children with ADHD work best with a qualitative over quantitative way of representing time. 
Exploring Movement Measure by an iPhone App. In the second phase insights were gained on body movement as a measure to predict the child's attention. Three children participated in a test using the 3-axis accelerometer of an iPhone with the application Accelerometer Data Pro [16]. A consent form was provided before the session allowing the researchers to observe and videotape the tests. Before the test children wore the iPhone for an hour and a half without measuring in order to get used to it. Each child worked independently for 20 minutes while wearing the smartphone measuring movement of the non-dominant hand. The data of all axes provided by the accelerometer is combined to come to one value of movement, allowing the plotted graph to be compared with the observed behavior.

Findings from the Explorative Stages. The comparison of the observations and the measured activity lead to a classification of three types of behavior: 1) On-task behavior (e.g. working as required by the teacher), is measurable by little movement, whereas 2) clear off-task behavior (e.g. when the child trows up his arms or acts goofy) is seen with larger amounts of movement above a threshold and 3) intermediate behavior involves an amount of movement larger than on-task but smaller than off-task behavior (Fig. 6). Task that seem useful at first, for instance, getting up to get a book, are often used by children with ADHD as a form of inattention and looking for stimuli. Such activitiess, can be distinguished by longer performance of movement and a more frequent occurrence within a certain timespan. Two children showed on-task behavior the first 8 minutes and performed more intermediate and off-task behavior later, whereas the third participant displayed regular intermediate and off-task behavior from the beginning. The results showed that an off-task behavior or larger amounts of intermediate behaviors are often the start of more and consecutive off-task behaviors, confirming literature [6], and demonstrating that returning to on-task work by themselves is difficult to do. The types of behavior classified matched the amount of movement in 25 instances, in only 4 instances it did not.

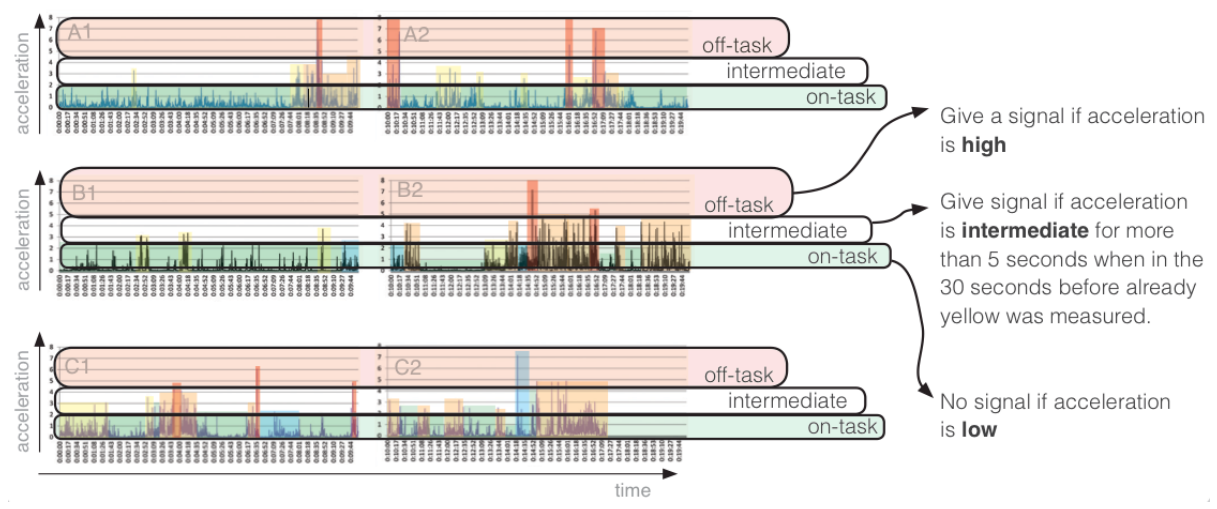

Fig. 6. Giving the signal at the right moment. Rules on when to give a signal based on movement and time 


\subsection{Final Concept and Prototype}

Therefore, WRISTWIT provides 4 visual feedback forms to support on-task behavior : a timer, a work signal, performance during class and score (Fig 7). When inattentive behavior is visible in an increased amount of restless body movements, the device provides feedback in the form of a work signal to get their attention back to work. The visual feedback is combined with vibration stimuli to make the child aware of their inattention.

To build up self-reflection moments and give children with ADHD the ability to link behavior to consequences, two additional forms of feedback are provided. Both are shown at the end of the class to ensure their attention to be on-task during class and create a motivating form of excitement. The performance of the class is shown at the end of each class by LEDs in the timer presenting a green, yellow and orange color-coding. It displays how the child behaved at which point in time: green for little movement and therefor attentive, yellow for less attentive and orange for even less. To keep the system positive red is not used (see Figure 7.c).

The amount of drop-shaped LEDs increases as the behavior is better. The drops, made up with 12 LEDs next to the LEDs from the timer, motivate the child to do better as it is something they desire to have: collect more of and share with others the results if wanted. The bracelet has a simple design that blends in with other bracelets children wear.

a)
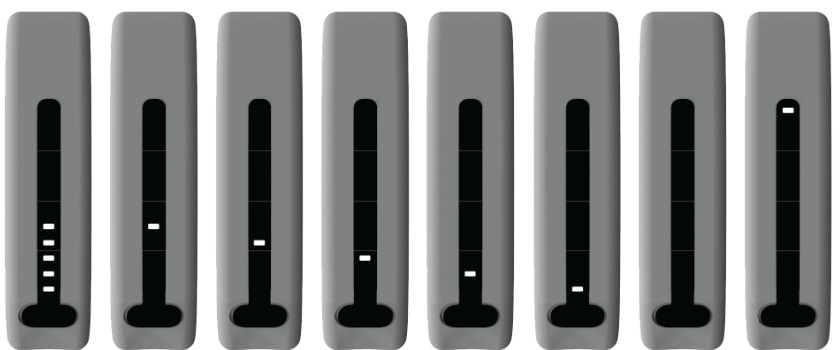

b)

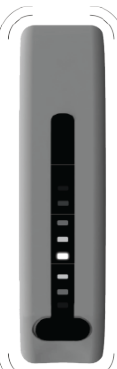

d)
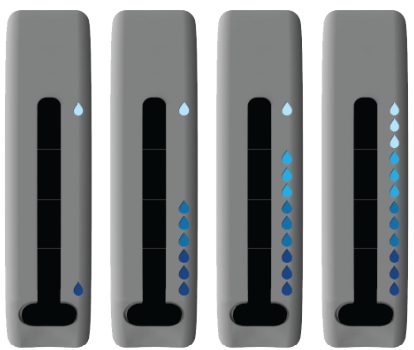

c)

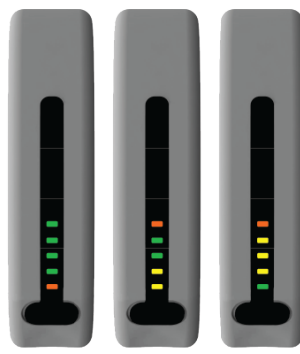

Fig. 7. WRISTWIT' feedback visualizations: a) timer b) worksignal c) performance d) score 
Later at home, the child connects the bracelet to the computer and the drops collected on the bracelet are translated to an amount of water to nurture a plant (Fig. 8a). The amount of water represents the score of that day, more water means better behavior; the child can compare today's performance to other days of the week (Fig 8b).
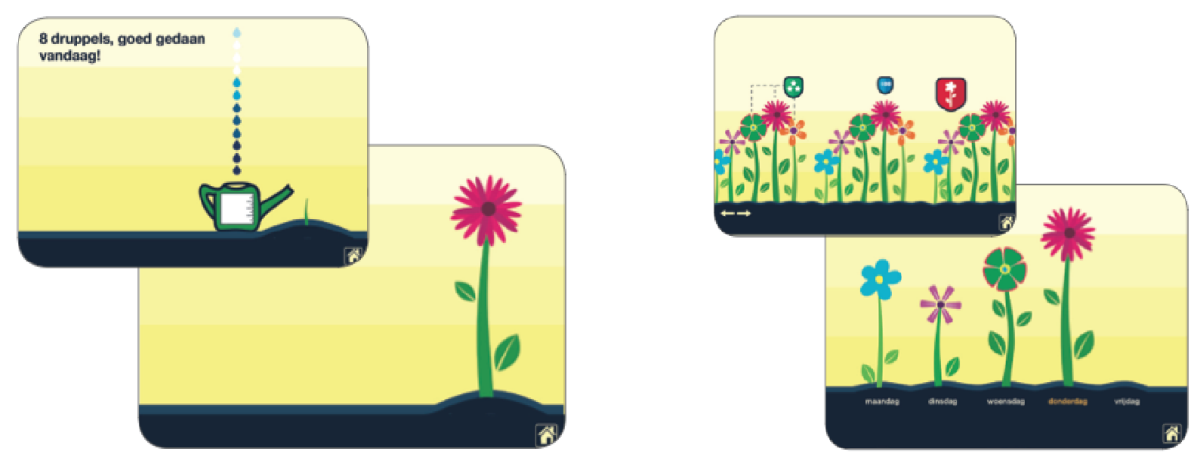

Fig. 8. WRISTWIT additional software. a) Left, today, drops grow a flower; b) right, comparison between days

\subsection{Final Test and Summary of the Results}

A semi-functioning prototype combined with Wizard of $\mathrm{Oz}$ method [17] was implemented to test the concept. The prototype was built using Arduino Uno, which included a color screen to represent the LEDs functionality (Fig. 8). This prototype comprises a $9 \mathrm{~V}$ battery and a vibration motor. The Wizard of $\mathrm{Oz}$ method allowed testing the functionality of the accelerometer to detect off-tasks behavior by simulating the vibration signal by means of an IR-receiver. A strap was implemented in the prototype to attach all parts and make it wearable during class time. The performance and the score are shown at the end of the class.

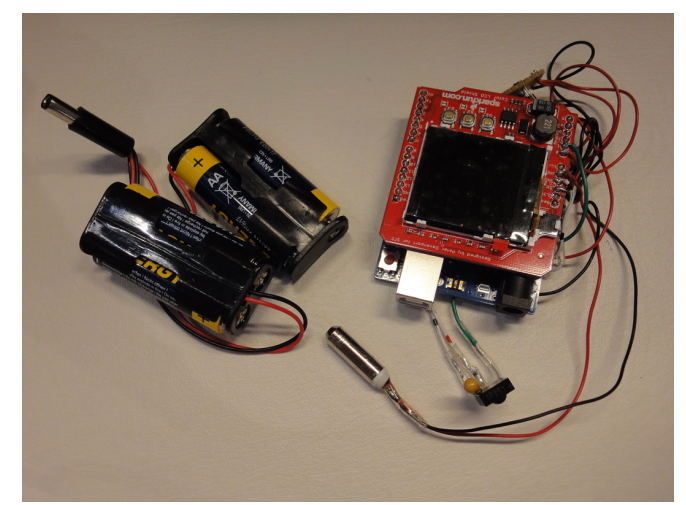

Fig. 9. Prototype components. Battery pack, Arduino One and vibration motor 
To evaluate how well children understood WRISTWIT and how much it was useful in supporting on-task behavior, a two days test was conducted with three children (Fig. 10). The parents signed a consent form. Participants were scheduled to use the prototype twice on the first day and three times on the second day, but due to limited testing time one child only tested twice in total. Each test lasted 20 minutes and took place in a class in which all children work independently. Only the basics of the prototype and its functionalities were explained beforehand. Based on the knowledge gained from observations with children, the researchers observed the behavior of the child and used the remote to give a work signal when the child was distracted. After each test a short interview allowed the researchers to get insights about the child's experiences with the device. A final interview checked their full understanding of the product. The material was analyzed and categorized for each function.

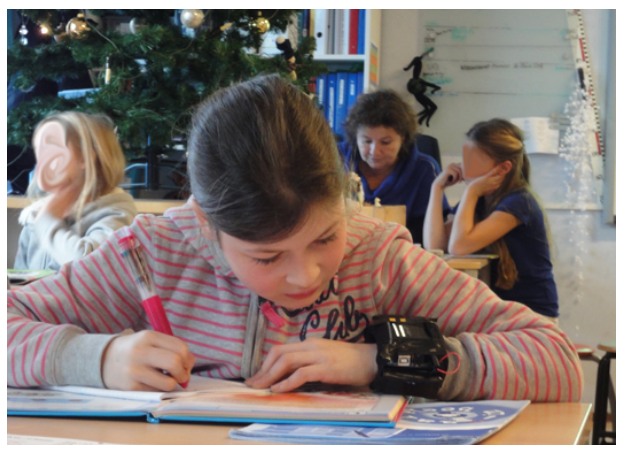

Fig. 10. Prototype in test

The results from the movement measurement study and the prototype test showed that it is possible to use movement as a measure for inattention. It was reported that each signal was understood by the children at first instance and elicited the intended reaction. Children compared their performance with their score supporting selfreflection processes. Difficulties with the prototype, giving unintended work signals due to signal communication failures, showed that children were aware of it reporting that was not fair, displaying more awareness of their own behavior. By simply wearing WRISTWIT and having the timer worked as a reminder. All children were committed to work as long as the timer was on. Interestingly, one of them continued during the break because the timer feedback had not been triggered yet.

Interviews conducted with teachers, parents and children after the tests gave insights on how children felt using the prototype. Children expressed feeling proud of collecting the drops, eager to get more and knowing what to do to improve. The personal character of the product was appreciated by all of them. Parents and teachers believed the personal character of the product motivates children more than other external motivation provided by them. Although the feedback signals of score and performance were found to motivate children to reflect on their personal results in order to improve their behavior, more extensive research is needed to see if these initial findings apply to a wider sample of participants and for longer periods of time. A larger study is also needed to understand how a drop in active behavior during class increases attention towards on-task behavior. 


\section{Discussion}

The design and evaluation of Personal Informatics solutions for ADHD children poses some challenges that are worth to discuss for the future development of KITA and WRISTWIT. The discussion addresses issues regarding the challenges of designing PI systems that go beyond the technicalities of collection, storage and data analysis and how these systems are conceived and tested in-situ.

\subsection{Supportive Role: Facilitating Reflection}

One of the biggest challenges in Personal Informatics is to provide meaningful information users can reflect upon in order to support self-awareness and selfreflection, to subsequently, encourage behaviour modification based on action. KITA and WRISTWIT share a common point: they support awareness and reflection by strengthening their roles on how the collected data from inertial sensors can be integrated and delivered to children with specific needs such as ADHD. As stated by [19], PI systems should be informative, effortless and simple to engage with. Low complexity interfaces have the potential to be more effective at motivating users in the long-term. KITA translates real-time physical behaviour data into a smiley face helping ADHD children to understand, in a way they had not thought about before, how their behaviour has short and long-term consequences. The progression of a bigger smile and awake/sleepy eyes facilitate self-monitoring progress in a friendly way. The use of a moody creature-like toy enabled children's level of engagement with the device. They stated that making his/her creature more and more happy was challenging and worth to try. On the other hand, WRISTWIT, provides data abstraction and visualizations facilitating the aspect of self-monitoring during ontasks behaviours at school. Not only by measuring child's inattention by assessing excess of body movement, but even more important to visualize time progression in a simple way, WRISTWIT gives the opportunity for children to self-monitor time on the go and sustained interest over time. Moreover, the haptic characteristic of KITA as a creature-like toy, and WRISTWIT as a bracelet offered opportunities to initiate an immediate engagement with children, inviting them to explore. A sensing-based interaction with meaningful information can engage children in a new way of thinking and it can fit their natural way of learning by exploring and playing with the material world.

\subsection{Personal Informatics in the Context: Designing and Testing In-situ}

The challenge of Personal Informatics is to design solutions that will succeed in becoming part of people's daily routines in their living context. In order to find directions to address this challenge, both prototypes were developed and tested insitu, with children, teachers and caregivers providing knowledge and personal preferences regarding ADHD treatment experiences. By means of several mock-ups and functional prototypes for both KITA and WRISTWIT, it was possible to explore what kind of information should be supplied that fits on the particular needs of 
ADHD children, as well as define when this information should be delivered based on the requirements of a specific context such as the school. The studies during class time provided insights of their use. We discuss that these insights of testing in-situ two proof-of-concepts bring forward a more direct and relevant design considerations for Personal Informatics targeting ADHD children. Considering design and testing insitu complements the role of KITA and WRISTWIT in addressing individual interest of 4-7 and 8-12 years old children facilitating their understanding of the provided feedback.

\section{Conclusions and Future Work}

Design and conceptualization process of the two prototypes discussed in this paper were derived from observations and interviews that took place in four schools. Witnessing class transitions between instruction, free and work time, exercise and play time provided information about how ADHD children behave during school, what they like to play, how teachers deal with burst of misbehaviors, as well as, information about time-awareness and constrains that a school setting could bring to children behavior. Next, in total 12 interviews with teachers, specialists, parents and children gave the possibility to pose questions that arose during observations. Researchers were interested in gaining insights about how children with ADHD experience rewards, restless periods, and time-awareness. Sensitizing booklets that enabled participants to provide more in-depth information followed these interviews. The material was analyzed and categorized in quotes and themes with concluding statements that helped researchers to define the design directions. First prototype iterations aimed to get insights into how body movement is a good measure to detect misbehavior and on/off-task behavior. In total 5 children participated in two tests. Two mock-ups, one designed for KITA using off-the-shelf accelerometer, and one more built specifically for WRISTWIT using an Arduino micro-controller. It is acknowledged that a higher number of testers are needed to be representative for a generalization in behavior change and self-awareness. However, the initial results open an interesting discussion about self-reflection in PI systems, it gave to the researchers rich insights about designing PI systems for a specific condition and context as ADHD, and it provided initial data that is possible to detect most of the behaviors right, and when the feedback signal can be given.

The design process of KITA and WRISTWIT was based on Empirical Research through Design process [14], User Centered Design methods and in-situ evaluations to inform the design of Personal Informatics devices for ADHD. The two experimental prototypes focused on integrating and visualize raw data of physical activity that fits in children's daily routines at school time. The design work demonstrates the potential for increasing children awareness with engaging feedback at the moment the child needs. ADHD behavior, reflected as activity patterns during the school day (KITA) or when time-based concentration is at risk, KITA and WRISTWIT, respectively, demonstrate the potential for ambient sensing technology and novel user-centered interfaces. 
Acknowledgments. We are very grateful to the schools De Bouwsteen Librijn, De Horizon, De Oostpoort in Delft, and De Elsenburgschool, in Rijswijk for their support and interest, as well as the specialists, teachers, parents and children who actively contributed to the development and tests of these projects.

\section{References}

1. Szatmari, P.: The epidemiology of attention- deficit hyperactivity disorders. In G. Weiss (Ed.), Attention-Deficit Hyperactivity Disorder. Vol 1 (pp. 361-371). Philadelphia, Pa: Saunders (1992)

2. National Institutes of Health [NIH]. National Institutes of Health consensus development conference statement: Diagnosis and treat- ment of Attention Deficit/Hyperactivity Disorder (ADHD). Journal of the American Academy of Child and Adolescent Psychiatry, 39, 182193 (2000)

3. Barkley, R.A.: Attention-Deficit/Hyperactivity Disorder. In Mash, E.J., Child Psychopathology (pp 75-143). New York: Guilford Press (2003)

4. Hallahan, D.P., Lloyd, J.W., Kauffman, J.M., Weiss, M.P., Martinez, E.A.: Learning Disabilities: Foundations, Characteristics and Effective Teaching, pp. 277-280 (2005)

5. GreatSchools.: Excerpts from his [Barkley's] lecture in San Francisco, CA on June 17, 2000. Retrieved April 6, 2012 (2008)

6. Fowler, M.: Increasing on-task performance for students with ADHD. New Jersey Education Association Review (2010)

7. AAPB, Applied Psychophysiology \& Biofeedback, http://www.aapb.org

8. Klingberg T., Fernell E., Olesen P., Johnson M., Gustafsson P., Dahlström K., Gillberg CG., Forssberg H., Westerberg H.: Computerized Training of Working Memory in Children with ADHD - a Randomized, Controlled, Trial. J American Academy of Child and Adolescent Psychiatry 44 (2):177-186 (2005)

9. Tryon Warren W., Shick T. G., Kazlausky T., Gruen W., Swanson J. M.: Reducing Hyperactivity with a Feedback Actigraph: Initial Findings, Clinical Child Psychology and Psychiatry (2006)

10.Yang, B., Chan, R.C.K., Zou, X., Jing, J., Mai, J., Li, J.: Time perception deficit in children with ADHD. Brain Research, Vol. 1170, pp. 90-96, doi:10.1016/j.brainres.2007.07.021 (2007)

11. Ian L., Anind D., Jodi F.: A stage-based model of personal informatics systems, Proceedings of the 28th international conference on Human factors in computing systems, April 10-15, Atlanta, Georgia, USA (2010)

12. Pirzadeh, A., Li He., Stolterman E.: Personal informatics and reflection: a critical examination of the nature of reflection. CHI Extended Abstracts 2013: 1979-1988

13. Munson, SA.: Mindfulness, Reflection, and Persuasion in Personal Informatics, Personal Informatics Workshop. CHI 2012. 6 (2012)

14. Keyson, D.V., Bruns, M.: Empirical research through design Proceedings And Conference Contributions. Empirical research through design. Proceeding of the Proceedings of the International Association of Societies of Design Research Conference (IASDR'09), 18-22 October 2009, Seoul, Korea, (pp. 4548-4557) (2009)

15. Visser, F. S., Stappers, P. J., Van der Lugt, R., \& Sanders, E. B.: Contextmapping: experiences from practice. CoDesign, 1(2), 119-149 (2005)

16.Wavefrontlabs, Accelerometer Data Pro, Retrieved October 27 (2012) 
17.Kelley, J. F.: An iterative design methodology for user- friendly natural language office information applications. ACM Trans. Inf. Syst. 2, 1 (Jan. 1984), 26-41. DOI= http://doi.acm.org/10.1145/357417.357420 (1984)

18.O'Malley, C. Fraser, D. "Literature Review in Learning with Tangible Technologies.” Nesta Futurelab series. Report 12, http://www.nesta-

futurelab.org/research/reviews/reviews_11_and12/12_01.htm (2005)

19. Burns, P. J., Christopher L., and Shlomo B.: Using Personal Informatics to Motivate Physical Activity: Could we be doing is wrong?." CHI 2012 Workshop. Vol. 1. (2012) 